\title{
Essais
}

Revue interdisciplinaire d'Humanités

Quels lieux pour les morts?

\section{Tourisme rouge, tourisme noir : le cas des memorial parks chinois}

Red tourism, black tourism: The case of chinese memorial parks

\section{Vincent Mariet}

\section{(2) OpenEdition \\ Journals}

Édition électronique

URL : https://journals.openedition.org/essais/8805

DOI : 10.4000/essais.8805

ISSN : 2276-0970

Éditeur

École doctorale Montaigne Humanités

Édition imprimée

Date de publication : 15 avril 2021

Pagination : 103-111

ISBN : 979-10-97024-09-3

ISSN : 2417-4211

Référence électronique

Vincent Mariet, "Tourisme rouge, tourisme noir : le cas des memorial parks chinois », Essais [En ligne], 17 | 2021, mis en ligne le 27 avril 2021, consulté le 18 janvier 2023. URL : http://

journals.openedition.org/essais/8805; DOI : https://doi.org/10.4000/essais.8805 


\section{Tourisme rouge, tourisme noir : le cas des memorial parks chinois}

Vincent Mariet

Depuis une trentaine d'années, le tourisme de mémoire se mue en un tourisme de masse. Investissant des « lieux historiques " ${ }^{1}$ anciennement touchés par la mort, il s'insère dans un processus global nommé thanatourisme ou "tourisme noir $"^{2}$. Dans le cas des lieux historiques où se sont déroulés des affrontements guerriers, si en Occident la question de la mise en tourisme de ces lieux de mort n'est pas nouvelle ${ }^{3}$, en Chine elle date d'une quinzaine d'années, le tourisme de mémoire étant englobé au sein du " tourisme rouge " depuis 2004, d'après la dénomination officielle utilisée par le Bureau central du Parti Communiste Chinois dans son document intitulé « Plan de développement national 2004-2010 du tourisme rouge $»^{4}$.

En Europe, le patrimoine lié à la guerre est considéré comme une « marchandise de tourisme de grande valeur ", un " produit prioritaire au potentiel touristique à développer "; tandis qu'en Occident de manière générale, il regroupe les "attractions touristiques les plus consommées " ${ }^{5}$. Cependant, une

1 En opposition aux " lieux de mémoire " de Pierre Nora et selon la qualification de Christine Pflüge citée dans l'avant-propos d'Hélène Camarade, «Lieux historiques, musées et mémoriaux ", in Camarade Hélène (éd.), "L’histoire par les lieux. Approche interdisciplinaire des espaces dédiés à la mémoire ", Essais. Revue interdisciplinaire d'Humanités, Bordeaux, École Doctorale Montaigne-Humanité, nº 6, 2014, p. 9.

2 Virgili Sandrine, Delacour Hélène, Bornarel Frédéric et al., "Des flammes à la lumière" : 100 ans de dark tourism autour du champ de bataille de Verdun ", Actes du Congrès, Metz, Université de Lorraine - laboratoire CEREFIGE, 2015, p. 2.

3 Ibid. Sur ce sujet, voir aussi l'article d'Anne Hertzog intitulé « Tourisme de mémoire et imaginaire touristique des champs de bataille ", Via [En ligne], n 1, 2012. http://journals.openedition.org/viatourism/1276 (consulté le 12 février 2019).

4 Alexeeva Olga, «La réinvention du passé : quel avenir pour les lieux de mémoire rouge dans la Chine d'aujourd'hui ?", Regards géopolitiques, vol. 2, n 1, printemps 2016, p. 21.

5 D'après Butler Richard et Suntikul Wantanee, Tourism and War, Abingdon-on-Thames, Routledge, 2012 ; Minic Natalija, "Development of "dark" tourism in the contemporary society ", Journal of the Geographical Institute Jovan Cvijic, n 62(3), 2012, p. 81-103 ; Smith Valene, "War and Tourism. An American Ethnography ». Annals of Tourism Research, n²5, 1998, p. 202-227. 
mise en perspective historique entre le tourisme de mémoire et la mort même, au travers de ses représentations et de la place dévolue ainsi à la mort et aux morts, n'a été que peu abordée dans le cas des cultures asiatiques ${ }^{6}$. En effet, les réflexions portant sur la mise en tourisme des lieux historiques liés à la guerre restent en grande partie issues de recherches anglo-saxonnes se focalisant principalement sur les champs de bataille du Vieux Continent. En ce qui concerne la Chine et son rapport à la dernière guerre mondiale, en décloisonnant notre regard hors de la sphère euro-occidentale, nous pouvons alors appréhender ce que plusieurs chercheurs anglo-saxons appellent, depuis le début des années 2010, le "souvenir transculturel " ${ }^{7}$. Cependant, le souvenir des morts n'est-il pas perverti par la transformation progressive, sous la poussée du tourisme de masse et de considérations politico-économiques, de lieux historiques de souffrances passées en véritables "parcs à thème " guerriers ? N'assisterait-on pas à une "disneylandisation ${ }^{8}$ programmée des espaces mémoriaux de l'Empire du Milieu?

En raison du faible nombre de recherches réalisées sur ce thème et encore moins sur l'espace chinois, cet article a ainsi pour objectif de défricher le sujet, en s'appuyant sur une étude de terrain réalisée à l'occasion de trois séjours effectués en Chine entre 2015 et 2020. À partir de trois sites visités dans la Municipalité de Shanghai et dans la province du Jiangsu (le Mémorial des martyrs de Longhua et de la bataille de Shanghai, le Mémorial des martyrs de Jiangyin et de la bataille du fleuve Yangzi, le Mémorial du massacre de Nanjing et du sac de la ville), il apparaît que la représentation de la mort en ces lieux s'inscrit dans un double discours politico-économique en parallèle d'un mimétisme mémoriel dit " universel ", voulu et entretenu par le PCC selon les caractéristiques chinoises.

\section{La Chine et le tourisme rouge}

En 2004, le Bureau central du PCC, en coordination avec celui du Conseil des affaires d'État de la République Populaire de Chine et celui de l'Administration nationale du Tourisme de Chine, a lancé un vaste programme de

6 Citons néanmoins Mickaël Lucken qui, dans son chapitre " Le tourisme historique ", aborde le cas japonais dans son ouvrage justement intitulé Les Japonais et la guerre 1937-1952, Paris, Fayard, 2013.

7 Graves Matthew et Rechniewski Elizabeth, "From Collective Memory to Transcultural Remembrance ", PORTAL : Journal of Multidisciplinary International Studies, vol. 7, $\mathrm{n}^{\circ}$ 1, janvier 2010. https://www.researchgate.net/publication/272731672_From_Collective_Memory_to_ Transcultural_Remembrance (consulté le 2 octobre 2019).

8 D'après la formule-choc de Renaud Ferrand, directeur de la Mémoire, du Patrimoine et des Archives au Ministère de la Défense, lancée lors du débat sur le "tourisme de mémoire " concernant les lieux historiques français liés à la Première Guerre mondiale, en amont du Salon mondial du Tourisme s'étant déroulé à Paris du 20 au 23 mars 2014. 
promotion du tourisme rouge pour dans un premier temps l'horizon 2010, reconduit en 2011 puis en 2017 avec vigueur. Ce plan d'action à long terme prévoit le développement systématique de ce type de tourisme au travers de centaines de lieux liés de près ou de loin à la Révolution chinoise et répartis dans toute la Chine?.

Le tourisme rouge, basé sur la mémoire collective spécifique à la Chine elle-même nommée " mémoire rouge ${ }^{10}$, a pour matrice la guerre civile chinoise (1927-1950), la Seconde Guerre sino-japonaise (1937-1945) et la construction de la "Nouvelle Chine " d'après la Seconde Guerre mondiale. En 1978, suite à la réouverture économique du pays sous l'impulsion de Deng Xiaoping et après de nombreuses disputes au sein du PCC, il est finalement décidé de mettre en valeur, par le biais du tourisme, de l'éducation et de la culture, l'héritage rouge d'essence maoïste. La croissance économique aidant, l'industrie touristique chinoise apparaît, depuis le milieu des années 1990, comme un domaine en pleine expansion où les secteurs publics et privés investissent de plus en plus massivement afin de satisfaire au mieux les touristes toujours plus nombreux ; les déplacements annuels pour le tourisme rouge étant évalués, d'après Anne Jaurès, à 800 millions pour l'année 2015, avec une progression annuelle de $15 \%{ }^{11}$. Le tourisme rouge représente ainsi aujourd'hui un quart du total des déplacements touristiques à l'intérieur de la Chine, et cet indéniable succès, fruit d'efforts engagés par les autorités tant sur le plan économique que sur le plan politique, repose en grande partie sur la jeunesse chinoise avide de nouvelles expériences étonnantes.

L'Administration nationale du Tourisme, ciblant notamment un public né à la fin du $\mathrm{XX}^{\mathrm{e}}$ siècle, a fait construire ou réaménager de nombreux lieux historiques et mémoriaux déjà existants en " "parcs à thème" dignes de Disneyland dans ces hauts lieux de lutte révolutionnaire " ${ }^{12}$. Dans ces "Martyr's Memorial Parks ", peuvent effectivement se côtoyer attractions traditionnelles (grande roue, tour panoramique) permettant d'apercevoir le site dans son ensemble ; expositions d'armements lourd (canons, obusiers) pour se représenter la ligne de front lors d'un parcours thématisé ; ou encore espaces de jeux vidéo (stratégie, infiltration, tir) au sein même du musée afin de pouvoir rejouer la bataille. En effet, dans l'Empire du Milieu actuel, la part de vidéoludisme dans l'esprit

9 Pei Yushen, Bourdeau Philippe (éds), «Le tourisme de mémoire en Chine : type Tourisme Rouge ", mémoire de master en Géographie, Aménagement, Environnement, Développement parcours Tourisme, Innovation, Transition, Grenoble, Université Grenoble-Alpes / Institut de Géographie Alpine, 2017, p. 7-10.

10 Ibid., p. 14.

11 Jaurès Anne, "Le tourisme rouge en Chine : véritable engouement ou carte forcée ? ", Monde chinois, $\mathrm{n}^{\circ} 29,2012$, p. 90-91.

12 Alexeeva Olga, «La réinvention du passé : quel avenir pour les lieux de mémoire rouge dans la Chine d'aujourd'hui ?", op. cit., p. 21. 
des jeunes chinois n'est pas à négliger car ayant une influence sérieuse quant à leur manière de percevoir la représentation de la mort dans des espaces originellement touchés par celle-ci et maintenant aménagés pour les loisirs ${ }^{13}$. Ainsi, sur des sites historiques liés à la Seconde Guerre mondiale, une scénographie "à l'américaine ", renvoyant autant aux jeux vidéo qu'au cinéma hollywoodien qui les ont inspirés, est en effet souvent mise en place.

Par conséquent, où se situe "la place des morts $"^{14}$ dans un tourisme de mémoire devenu un "produit touristique de la culture et de l'esprit " ? $^{15}$ Comment peut-elle être en adéquation avec le développement planifié d'un territoire ? L'observation et l'étude de lieux conceptualisés, agencés et transformés, dans l'espace culturel chinois, permettent de montrer comment peut être représentée la mort selon des codes propres à l'entertainment.

\section{De lieux sacralisés aux espaces de divertissement}

Dans la Chine contemporaine, la représentation de la mort, notamment due à la guerre, s'apparente à l'une des formes ou expressions du patriotisme. De nature laïque, elle reste néanmoins indissociable de la pensée chinoise où la mort est considérée comme la transformation du qi de l'être vivant, qui s'inscrit donc dans la continuité de la vie elle-même ${ }^{16}$. Symboliser la disparition dans des lieux sacralisés renvoyant à des périodes historiques sombres vécues par le pays est alors un des moyens mis en œuvre pour représenter la mort.

Au mémorial de Nanjing, espace éminemment sacralisé où l'on se doit d'être silencieux et de faire preuve de respect envers les morts, une certaine distanciation est appliquée lors de la mise en place du parcours de visite. Le suivi d'un chemin balisé, tracé à moyenne distance du " mur du souvenir » ou encore des charniers, permet ainsi de laisser libre champ aux âmes des morts - dans ce "vide primordial " d'inspiration taoïste ${ }^{17}$. Si l'importance de la géomancie dans la culture chinoise amène à considérer sérieusement la disposition du site-mémorial selon des préceptes de nature philosophique, l'athéisme prôné par le PCC l'aligne en parallèle sur des références mondialisées d'inspiration occidentale pouvant somme toute paraître paradoxales.

13 La Chine se place maintenant première en nombre de joueurs et en termes de revenus liés aux jeux vidéo : "Top 100 Countries by Game Revenues », Newzoo [En ligne]. https://newzoo. com/insights/rankings/top-10-countries-by-game-revenues/ (consulté le 19 février 2019); "L'industrie du jeu vidéo en Chine », Indie Game Factory [En ligne]. https://indie-gamefactory.eu/lindustrie-du-jeu-video-en-chine (consulté le 3 septembre 2019).

14 D'après le titre de l'ouvrage de Patrick Baudry, La place des morts. Enjeux et rites, Paris, L'Harmattan, coll. « Nouvelles études anthropologiques », 2006.

15 Pei Yushen, Bourdeau Philippe (éds), "Le tourisme de mémoire en Chine : type Tourisme Rouge ", op. cit., p. 8.

16 Cheng Anne, Histoire de la pensée chinoise, Paris, Seuil, 1999, p. 454.

17 Margat Claude, L'Échappée chinoise, Paris, Éditions du Canoë, 2019, p. 157. 
Ainsi, la monumentalisation du mémorial de Nanjing est couplée à une artialisation puisant dans des repères universels et normatifs, à l'image de la croix chrétienne, vidée de sa substance religieuse sans pour autant être reniée et qui devient par conséquent un point de repère de l'espace de mort. Au sein de ce dernier, au fil du cheminement, le rapport à l'invisible étant primordial dans la pensée chinoise, l'échange symbolique entre les vivants et les morts s'effectue donc par le biais d'une mise en scène de l'invisible, permettant de « retenir les morts » vivant dans « le monde invisible», maintenus par un «flux vital » comme celui « qui d'année en année fait revenir la végétation ${ }^{18}$. De fait, la symbolique des plantes y est particulièrement prégnante. Que ce soit à Nanjing ou à Shanghai, le " paysage allégorique de la mort " ${ }^{19}$ y est matérialisé par un chemin encadré de bambous débouchant sur un ancien lieu de mort ; à Nanjing le charnier dit "des 10000 cadavres ", à Shanghai le lieu d'exécution des condamnés près du temple de Longhua. Parmi les nombreuses symboliques attribuées au bambou, celle de la résilience corollaire de la paix après la guerre est en effet un exemple d'harmonie retrouvée entre le monde naturel et l'humain, la mort et la vie, selon le principe du yin et du yang. Aux côtés des bambous, sont aussi très présentes, dans les memorial parks, les fleurs de lotus, symbolisant la vitalité et les âmes réincarnées purifiées tandis que les chrysanthèmes jaunes évoquent le renouveau et la vie qui se poursuit en des lieux meurtris. Enfin, la représentation de la mort sacralisée se fait également par la disposition d'une flamme en souvenir des morts, des « martyrs de la Révolution ", au sein du site-mémorial.

Cependant, en ayant comme objectif de promouvoir une narration transnationale de la mort due à la guerre à destination de touristes-consommateurs, les administrateurs chinois ont ainsi lancé la création de nouveaux concepts. Au même titre que d'anciens abattoirs et installations industrielles sont transformés en centres commerciaux d'attractions à Shanghai ${ }^{20}$, les sites-mémoriaux sont eux aussi aménagés de telle sorte qu'ils puissent s'intégrer dans leur territoire en tant que lieu de divertissement ouvert faisant partie intégrante de l'espace public. En prenant l'exemple des memorial parks de Jiangyin dans le Jiangsu et de Longhua à Shanghai, l'un semi-naturel le long du fleuve Yangzi, l'autre entièrement urbain dans le district de Xuhui, il apparaît que la création de parcours et autres circuits ouvre l'ensemble du site à la totalité de

18 Javary Cyrille, La souplesse du dragon. Les fondamentaux de la culture chinoise, Paris, Albin Michel, coll. «Espaces libres », 2017, p. 64-68.

19 Jullien François, «La conception du monde naturel, en Chine et en Occident, selon Tang Junyi (La valeur de l'esprit de la culture chinoise) ", Extrême-Orient, Extrême-Occident, n 3, 1983, p. $117-125$.

20 À l'image du " 1933 Old Mill Fun ", abattoirs-usine d'avant-guerre et espace de mort reconverti en centre commercial regroupant restaurants et boutiques, manèges et attractions, bureau du tourisme et musée d'histoire, etc. 
l'espace constituant le « champ de bataille » ou la " carte de guerre »; espace de mort dans le passé, espace de vie dans le présent. Ceci entraîne l'émergence de nouvelles représentations et usages des mémoriaux, qui deviennent des lieux d'hybridation des pratiques ${ }^{21}$. La mise en valeur des champs de bataille consiste par conséquent à les transformer en "lieu d'histoire " (aménagement de chemins, installation de panneaux explicatifs), en "lieu de mémoire " (mise en avant des ruines, restauration de monuments), en "lieu de randonnée " (voies vertes, parcours sportifs) et en "lieu d'exposition artistique " (classique et militaire, contemporaine et universaliste). Au sein de ce large espace, la scénographie mise en place tout au long du parcours de visite du site-mémorial ainsi que l'organisation de cet espace s'organisent généralement selon l'ordre suivant : le parc extérieur, puis le lieu historique en tant que tel, et enfin le musée. Hugo Remark, considérant les espaces muséographiques comme des

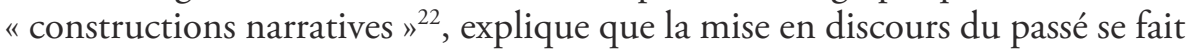
selon les mêmes principes que celle du récit de fiction. En Chine, la mise en scène qui l'accompagne, issue d'une volonté politico-économique selon un modèle de développement planifié, se matérialise donc sous la forme de ces memorial parks, intégrés à l'espace public; ces parcs où l'on nous met sur les traces des "morts en martyr ", que ce soit à Nanjing, au parc Huangshan à Jiangyin, ou en suivant le " chemin rouge " à Shanghai ${ }^{23}$.

La mise en divertissement de ces lieux particuliers, de plus en plus investis par le grand public, s'accompagne du développement d'un tourisme singulier qui s'appuie sur la visite de ces espaces touchés et/ou dévolus à la mort et où celle-ci, du fait de l'imaginaire et de la pensée inconsciente des jeunes contemporains fortement influencés par la gamification quant à leur vision de la guerre, peut y être représentée de manière ludique et spectaculaire.

\section{Entre thanatourisme et ludification}

Si l'invention d'une politique touristique et culturelle autour de la guerre répond à des enjeux d'aménagement du territoire, la construction d'un memorial park est pour sa part un des moyens utilisés pour le valoriser. En effet, les fonctions culturelles diversifiées du mémorial (musée, centre de recherche, lieux d'exposition et de concert, etc.) en font ainsi un outil d'aménagement privilégié destiné à équilibrer l'offre touristique dans un territoire en marge.

21 Hertzog Anne, "Tourisme de mémoire et imaginaire touristique des champs de bataille », op. cit., p. 12.

22 Remark Hugo, "Les expositions d'histoire comme constructions narratives ", in Camarade Hélène (éd.), " L'histoire par les lieux. Approche interdisciplinaire des espaces dédiés à la mémoire ", Essais. Revue interdisciplinaire d'Humanités, Bordeaux, École Doctorale MontaigneHumanité, n 6, 2014, p. 55-67.

23 "Découverte du chemin rouge ", Meet in Shanghai [En ligne]. http://fra.meet-in-shanghai. net/the_red_tours.php (consulté le 4 novembre 2019). 
Sur les nombreux anciens champs de bataille reconvertis que compte le territoire chinois, un nouveau tourisme se fait par conséquent jour : le thanatourisme. Ce tourisme particulier, qui se bâtit sur la base de catastrophes de tous types et donc des morts dues aux guerres, mêle deux tendances : la mondialisation touristique dans son ensemble et les particularismes d'enjeux locaux de développement du territoire ${ }^{24}$. Ainsi, la mise en divertissement d'un espace touché par la mort pour en faire une destination désirable impose de s'interroger sur les représentations de la mort associées aux violences de guerre ainsi qu'aux registres utilisés permettant de les rendre acceptables. Il faut en effet, en premier lieu, donner de quoi voir d'impressionnant au grand public $^{25}$. Les processus d'exhumation des traces et le marquage ou la délimitation des lieux de conflit doivent conférer de la visibilité à des éléments que l'on ne peut appréhender immédiatement puisque appartenant au passé. De ce fait, la reconstitution historique en constitue une modalité particulière puisque instituant un semblant de vérité à travers des mises en scène pseudoréalistes et spectaculaires ${ }^{26}$. La théâtralisation chinoise de la mort guerrière se matérialise par des bas-reliefs représentant des figures humaines ou encore par d'imposantes sculptures d'art réaliste d'hommes et de femmes couleurs grise et carmin s'effondrant sous les balles. Cette narration visuelle de la mort, dramatique et tape-à-l'œil, donne un cachet cinématographique à la scénographie du parc mise en place sur le chemin du visiteur. La reconstitution et/ou la représentation de la bataille passée passe ainsi par exemple à Jiangyin, par la réinstallation dans les bunkers d'origine de canons tout au long du parcours ; à Shanghai, par l'installation d'un corps brisé monumental allongé en position latérale sur le sol ; ou à Nanjing, par la représentation en pierre blanche d'un corps déchiqueté. Dans ce dernier mémorial, la mise en lumière des squelettes issus des historiques charniers redécouverts dans les années 1980 ajoute à la spectacularisation morbide du lieu. La pédagogie est ainsi nettement mise de côté, pour ne garder que la mise en scène grandiose, qu'elle soit classique ou modernisée au moyen de nouvelles technologies (hologrammes, écrans incurvés, cinémas $4 \mathrm{D}$ et à $360^{\circ}$, etc.) toujours plus immersives.

Les administrations chinoises en charge de développer le tourisme utilisent les mêmes ressorts que ceux de l'ouverture économique. C'est pourquoi, dans le but d'attirer un public urbain jeune et curieux, la délimitation d'enclaves territoriales mémorielles s'accompagne bien souvent de la construction de circuits touristiques ponctués de "parcs à thème $"^{27}$. Le mémorial de Longhua

24 Folio Fabrice, «Dark tourism ou tourisme mémoriel symbolique?», Téoros [En ligne], n 35(1), 2016, p. 1. http://journals.openedition.org/teoros/2862 (consulté le 4 novembre 2019).

25 Hertzog, Anne, "Tourisme de mémoire et imaginaire touristique des champs de bataille ", op. cit., p. 12.

26 Ibid.

27 Ged Françoise, «Shanghai : du patrimoine identitaire au décor touristique. Le laboratoire de 
est particulièrement représentatif de la mise en divertissement d'un ancien espace de mort par le biais d'attractions, installées au sein même du mausolée : bâtiments néo-futuristes de formes pyramidales et sphériques, diffusion de musique dans les allées et les tunnels, café-restaurant et espaces jeux vidéo dans le bâtiment-mémorial. Dans les espaces muséographiques, le visiteur du parc lui-même est invité à devenir joueur et à tuer les différents agresseurs de la Chine des années 1930-1940 jusqu'à ce que sa propre mort éventuelle marque le game over. Cet exemple éloquent montre comment la reconversion d'espaces originellement dévolus à la sacralisation de la mort suppose des pratiques socio-spatiales innovantes. Les memorial parks, tenant à la fois du musée patriotique de plein air et du parc d'attractions, en sont donc ainsi la parfaite illustration.

Lieux de consommation du patrimoine historique et militaire chinois, ces parcs de loisirs d'un genre nouveau contribuent donc fortement au renouvellement de la popularité, auprès des jeunes touristes intérieurs du pays, d'espaces mémoriels alors vus comme figés et où se trouve maintenant représentée la mort de manière cinématographique ou ludique à visée divertissante ; espaces rarement visités par les Occidentaux, ces derniers étant plutôt en quête d'une Chine culturellement authentique, immuable et paisible ${ }^{28} \ldots$

\section{Conclusion}

L'objectif de cette recherche est d'étudier l'évolution de la mise en tourisme de sites meurtris particuliers, soit trois champs de bataille situés dans la Municipalité de Shanghai et dans la province du Jiangsu, sur ces trente dernières années. Les résultats de cette recherche mettent en évidence la manière dont l'offre de tourisme s'est structurée dans le temps, opérant progressivement une mise en tourisme tournant autour d'un récit de fiction n'ayant plus de contenu politique offensif à proprement parler mais conservant toujours cette description romantique du passé révolutionnaire et de ses morts en martyr. Progressivement, à mesure que la distance temporelle croît par rapport à l'événement - la bataille en son lieu - la vision de la mort devient plus claire et sa représentation peut alors entrer dans une phase de ludification s'insérant dans un processus de thanatourisme. Ouverte à un renouvellement qui la déleste progressivement de certaines contraintes originelles, le changement générationnel et les nouvelles technologies aidant, la mort n’est donc plus un frein mais un tremplin à l'entertainment. Nous observons ainsi le

la nouvelle Chine ", Les Annales de la recherche urbaine, $\mathrm{n}^{\circ} 72,1996$, p. 79-88 ; Duthion Brice, Walker Lionel, Les patrimoines touristiques naturels, historiques, culturels, Paris, De Boeck, coll. "Tourisme compétences \& métiers », 2015, p. 46.

28 Voir David Béatrice, "Tourisme et politique, la sacralisation touristique de la nation chinoise ", Hérodote, $\mathrm{n}^{\circ} 125,2007 / 2$, p. 143-156. 
passage du tourisme de mémoire au tourisme de divertissement, pour arriver à une tension entre la sacralisation et le loisir au sein de parcs d'attractions, qui se placent à mi-chemin entre le tourisme rouge et le tourisme noir.

\author{
Vincent Mariet \\ UMR 7266 LIENSs \\ Université de La Rochelle \\ vincent.mariet1@univ-lr.fr
}

\title{
Résumé
}

Depuis une trentaine d'années, le tourisme de mémoire se mue en un tourisme de masse. En Chine, il se dilue dans un " tourisme rouge " officiel qui lui-même s'insère dans un processus global de thanatourisme. Les anciens champs de bataille, espaces mémoriels sacralisés, sont maintenant le théâtre d'une mise en divertissement sous la forme de memorial parks. Cet article a pour objectif de montrer comment et par quels moyens est représentée la mort due à la guerre et quelle est alors la place des morts dans ces lieux historiques au passé sombre.

\section{Mots-clés}

Tourisme rouge, thanatourisme, sacralisation, ludification, memorial parks.

\begin{abstract}
Over the last thirty years, remembrance tourism has become mass tourism. In China, it is being diluted in an official "red tourism" which is itself a part of a global process of thanatourism. The former battlefields, sacred memorial spaces, are now the scene of entertainment taking the form of memorial parks. The aim of this article is to show how and by which ways death due to war is represented and which place the dead have in these historical places concerned by a dark past.
\end{abstract}

\section{Keywords}

Red tourism, Dark tourism, sacralisation, gamification, memorial parks. 